\title{
An Application to Train Eye Movement Control among Thai Juniors
}

\author{
Inthraporn Aranyanak ${ }^{+}$
}

School of Science, King Mongkut's Institute of Technology Ladkrabang, Bangkok, Thailand

\begin{abstract}
Reading is an important skill for acquiring knowledge throughout life. Without the ability to read fluently, opportunities for personal fulfilment and career achievements will be decreased. This study developed an application exploiting the concept of a word's optimal viewing position (OVP) with a gaming design to encourage students to read and practice their eye movement control to recognise a word quickly. A pilot study was conducted by training grade 3 students with the application with the OVP to guide their eye fixation for 7 days to explore if their reading performance improved. Before and after training sessions were conducted without using the OVP. The result shows that those students have increased their reading performance approximately $61 \%$ after the training sessions without the OVP and approximately $64 \%$ with the OVP guide. The optimal viewing position game helps the readers recognise words faster. Quantitative data were collected using Net Promoter Score (NPS). The finding shows the application has an NPS score of 33 indicating that the application is doing good and has more happy users than unhappy ones. Qualitative data was also collected by interview and found that the children were satisfied with the interface design, the application is easy to use, but they were not familiar with some words causing them to be mispronounced.
\end{abstract}

Keywords: educational technology, children reading game, optimal viewing position

\section{Introduction}

Literacy is a key factor in determining the quality of our life and career opportunities. Thai government declared reading as a national issue that must be seriously promoted since 2009. In Thailand, primary education begins at the age of 6. From the basic education core curriculum of Thai language in 2008, by grade three, children should be able to understand and summarise insights from reading literary works for application in daily life; express views from the literature and literary works read; and have good reading skills [1]. Even though $96 \%$ of Thai population aged 15 years and above are literate [2], Thailand's reading performance in PISA 2018 was lower than in any previous assessment; ranked $66^{\text {th }}$ among 79 countries [3]. Improving Thai children's reading performance is one of the Thai government's goals. Thailand has spent significant amounts of money on education. Unfortunately, the students of Thailand still scored below global averages in key subjects in various international tests. This is due to their low efficiency in reading skill influencing on academic performance. Therefore, if children can read fluently, it is a good sign that they understand what they're reading. That's why reading fluency is one of the measures schools use to track progress as children learn how to read.

During reading, people do not move their eyes smoothly but jump from one location to another with considerable variation in latency between movements. When the eyes move rapidly it is called a saccade which lasts for 20-40 ms. Readers acquire information from the text during eye fixations. The average fixation duration is 200-250 ms. An average reading speed in junior level is between 53-107 words per minute (wpm) though some readers are naturally quicker than others [4]. The effect of optimal viewing position (OVP) has been extensively studied for years especially in the research on visual word recognition. Previous studies have shown that fixating eyes at the OVP results in shorter recognition times $[5,6]$.

The purpose of this study is to develop an application for training readers to control their eye movements during reading so that the saccades they make land at the center of the word (at the OVP). This will help Thai students in junior primary schools to improve their reading speed. Hence, if they can recognise a word quickly, it will enhance their overall reading speed and contribute positively to their academic performance.

\footnotetext{
+ Corresponding author. Tel.: + 662-329-8220.

E-mail address: Inthraporn.ar@kmitl.ac.th.
} 


\section{Background and Related Work}

\subsection{Thai Script}

The Thai alphabet is derived from the Old Khmer script. It was modified and introduced tone marks. Thai alphabet consists of 44 consonant symbols and 32 vowel forms and four tone markers. The characters are written from left to right in horizontal lines. There are two different kinds of vowels, short and long vowels. Some vowels are written horizontally along with the consonants; initial or final position of the consonants, but some are written vertically above or below consonant characters. All tone markers are located above the initial consonant alphabet. In Thai writing, there is no space between the words in a sentence, nor after a full stop at the end of a sentence. In general, it can be used when finishing a phrase, clause or sentence to start a new idea, or between a series or set of words or phrases, or before and after a person's title and name, etc. [7]. Moreover, a space character may appear within a sentence for linguistic or prescriptive orthographic reasons [8]. Therefore, sometimes it may lead to ambiguity on word segmentation, such as, "รถทับสุนัขตาย" The sentence can be interpreted either "A car ran over a dog, the dog was dead" or "A car ran over a dog, the dog is dead".

\subsection{The Optimal Viewing Position (OVP)}

The optimal viewing position is a common phenomenon in word recognition studies. Readers can identify words more quickly and less refixations are needed on words when the first eye fixation lands close to the center of the word. Displaying words such that the optimal viewing-position (OVP), a location between the beginning and middle of a word, lies at the centre of a fixation increases reading speed in individuals with normal vision [6]. An eye movement study showed that Thai children's eye movement patterns were similar to adults, where the preferred viewing location (PVL) for readers is just before the middle of the word [9]. Lehtimäki and Reilly (2005), described a study to design and evaluate an oculomotor reading aid for beginning readers. The OVP game was developed for training young readers to control their eye movements during reading. The authors used an eye tracker to capture readers' eye movements in real time and give them feedback about their fixation location. The study demonstrated that the training game had the desired effect on the landing site distribution, where the peak of the landing site distribution moved towards the OVP in most participants. As a result, when readers move their eyes and fixate on the OVP they achieve better reading performance [10].

\section{Methods}

\subsection{Application}

The application was developed using Java on Android Studio and the data were connected to Firebase. The application can be installed on android mobile devices. It was designed to be children friendly; colorful, using easy language, and amusing background sound effects.
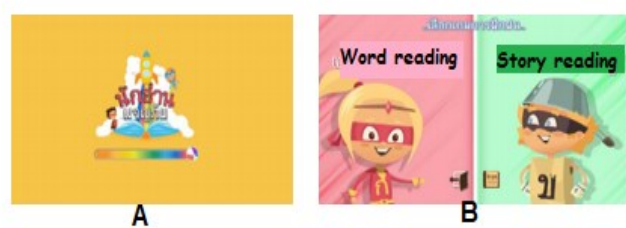
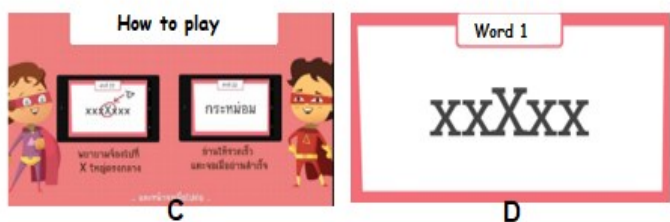

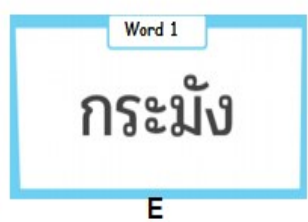

Fig. 1: The above pictures show example interfaces of the word reading part

Once the application is loaded as shown in Fig. 1-A, a user needs to register their demographic information then login to start the game. The application consists of two parts as shown in Fig. 1-B; word reading and story reading. When the word reading part is selected there are two types of the game available: one without the OVP guide, another one with the OVP guide used in training sessions. Once word reading with the OVP is selected, a brief explanation of the game is displayed as shown in Fig. 1-C. Fig. 1-D presents the X line for training students to fixate their eyes on the OVP of the word. The screen is displayed for three seconds then a training word is shown at the same location of the $\mathrm{X}$ line as shown in Fig. 1-E. Then, a user says the word out loud as soon as possible. 

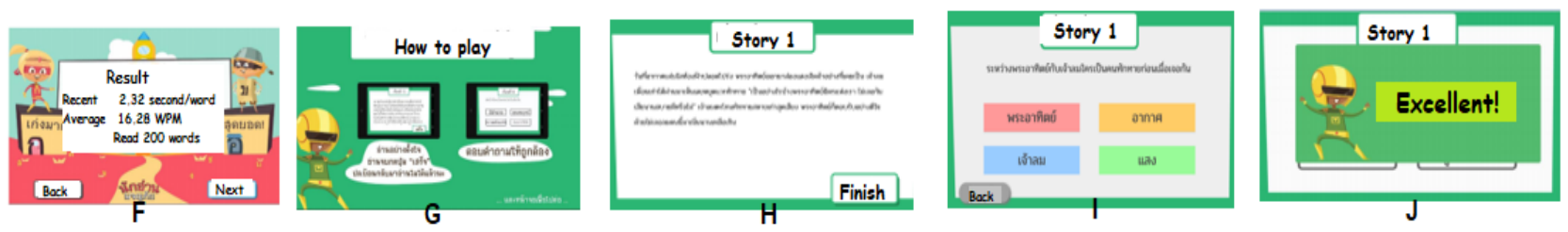

Fig. 2: The above pictures show example interfaces of the story reading part.

Response times are recorded for word-per-minute calculations and shown every 20 words as shown in Fig. 2-F. Fig. 2-G represents the screen of instructions on how to play with the story reading part. In this part, a short story is displayed on the screen as shown in Fig. 2-H. Once a reader finishes reading and presses the finish button, Fig. 2-I will be shown to ask a question with four choices for checking reading comprehension. If the reader answers correctly, Fig. 2-J is displayed for reinforcing compliments, such as, "Good job", "Excellent".

\subsection{Materials}

\section{- Word reading part}

Words were selected from a website called Trueplookpanya which contains a Thai word corpus on the website. One thousand two hundred and six words used in grade 3 were selected from the corpus. Two hundred words were randomly picked from the dataset for before and after the training sessions and also during each training session. Each session contains words of length between 4 and 8 characters; 40 words of length 4,80 words of length $5-6$, and another 80 words of length 7-8.

\section{- Story reading part}

In this part, sentences for a comprehensive reading were chosen from the baby.kapook.com website. The website provides many stories for Thai juniors. Thirty short stories, each containing between 75 to 90 words, were selected from the website. After presenting each story on the screen, there was a question with four choices to check the readers' understanding.

\subsection{Pilot Study}

The pilot study was conducted at Wittayanont school, Bangkok. Two Grade 3 participants, one boy and one girl, aged 8, were recruited into the pilot study (see Fig. 3). Only the word reading part was employed in the study. The readers' eye movements were trained to fixate on the OVP using the application over a period of seven days. The training sessions lasted 20 minutes. Data from the day before and the day after the training sessions were also collected without the OVP guide. The participants were rewarded with a small gift after the study. The program was run on a notebook instead of a tablet since the tablet had proved unreliable while collecting data from the pilot study. The application was run on LDplayer which is free software for the Android gaming emulator for PC. The notebook screen size is 14 inches (1920x1080); Acer Swift 3 SF31451 57TR; Windows 10 Pro.
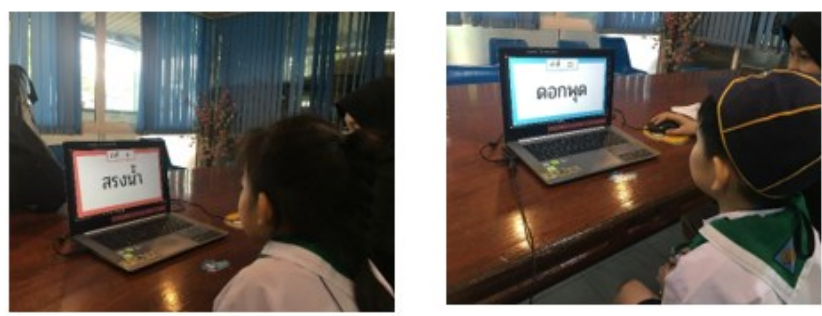

Fig. 3: Two students on Grade three were training their eye fixations on the OVP.

Steps to conduct the pilot study are as follows:

1. Word reading without the OVP guide containing 200 words was given the day before treatment sessions. Each word was displayed in the center of the screen. The participants were asked to read aloud as soon as they saw the word. There was a break every 20 words.

2. The training sessions occurred for 7 days. The $X$ line was shown in the center to help the students fixated their eyes on the OVP for 3 seconds then an actual word was displayed on the screen. The participants were asked to read aloud as soon as they saw the word. There was a break every 20 words. 
3. Word reading without the OVP guide was given again on the last day and a Net Promoter Score (NPS) survey question was asked to measure users' satisfaction with the application followed by an interview.

\section{Results}

The application was evaluated using NPS. In user experience research, NPS is used to measure customer loyalty, satisfaction, and enthusiasm with a company that is calculated by asking customers one question "how likely are you to recommend this product to a friend or colleague?" rating on a scale from 0 to 10 . The result shows that $0 \%$ of respondents are Detractors, $67 \%$ are Passives and $33 \%$ are Promoters, given the NPS score is 33 indicating your product is doing good and has far more happy users than unhappy ones. An interview and data analysis reported that the children were satisfied with the interface design, the application is easy to use, but they are not familiar with some words causing them mispronouncing.

Table 1: A comparison of reading performance before and after the training sessions in words per minute (WPM)

\begin{tabular}{|l|c|c|c|c|}
\hline \multirow{2}{*}{ Gender } & \multicolumn{2}{|c|}{ Before training } & \multicolumn{2}{c|}{ After training } \\
\hline & $\begin{array}{c}\text { Time } \\
(\mathrm{min})\end{array}$ & $\begin{array}{c}\text { Speed } \\
(\mathrm{WPM})\end{array}$ & $\begin{array}{c}\text { Time } \\
(\mathrm{min})\end{array}$ & $\begin{array}{c}\text { Speed } \\
(\mathrm{WPM})\end{array}$ \\
\hline Girl & 19.89 & 10.05 & 11.80 & 16.95 \\
\hline Boy & 17.98 & 11.12 & 11.68 & 17.11 \\
\hline Average & 18.94 & 10.59 & 11.74 & 17.02 \\
\hline
\end{tabular}

From the pilot study, reading performance before and after the training sessions on the word reading part; without the OVP guide are presented in Table 1. An average reading speed before training eye movement controls on the OVP is about 11 words per minute while after the training sessions an average of reading speed is up to 17 words per minute. The reading performance increases $60.72 \%$. After the training sessions, the students spent less time on the word reading recognition.

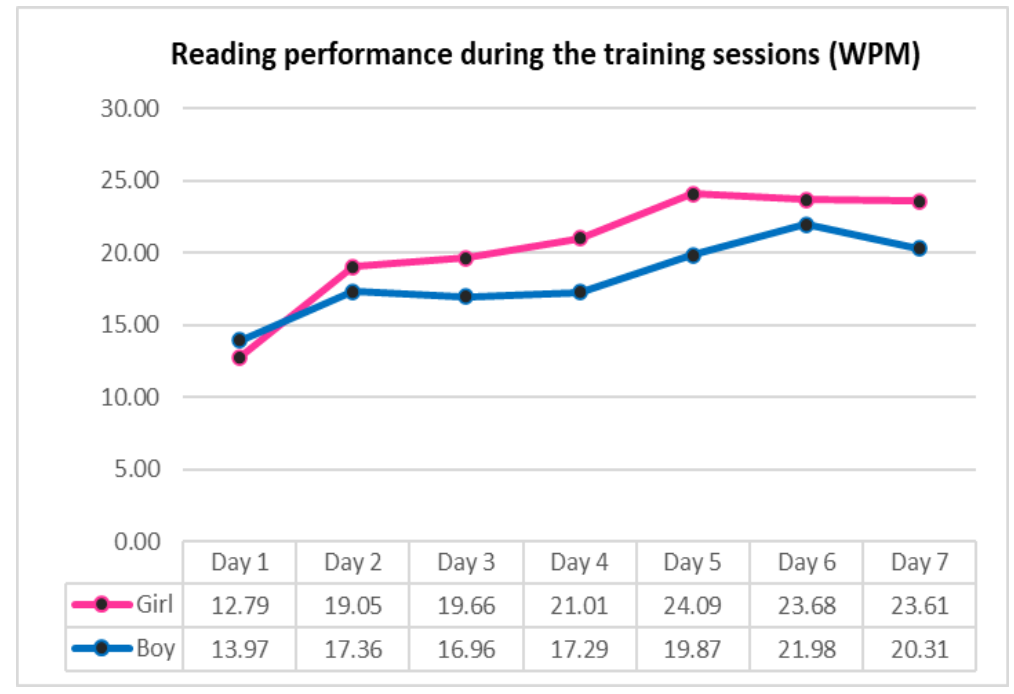

Fig. 4: Reading performance during the training sessions of the Thai students in a grade 3 level.

Fig. 4 displays reading speed in words per minute during the training sessions for 7 days. The pink line represents the girls' reading performance and the blue line represents the boys' reading performance. Both lines gradually rise up over time. This may imply that when readers fixate their eyes on the OVP, their word reading recognition improves. The reading performance comparing between day 1 and day 7 increases $64.12 \%$.

\section{Conclusion and Future Work}

This paper presents a reading mobile application for Thai students on junior levels. The system was developed on Android and using the Firebase backend service. The application was designed to be a user friendly for children using colorful, easy language, amusement and background sound effects. It consists of two parts; word reading and story reading parts. For the training sessions in the word reading part, it is used 
to train children's eye movement to fixate on the optimal viewing position (OVP), which is located at the center of the word or just left of it. The phenomenon was found in many visual word recognition research indicating where words are recognised faster than other locations. In the story reading part, it is for reading short stories. Each story is followed by a comprehensive question with multiple choices to check readers' understanding. A pilot study was conducted with two students on grade 3 in a primary school. Only the word reading part was employed for training their eye movement control. They were in the training session for 7 days. Word reading without the OVP was examined before and after the training sessions to compare their reading performances. The finding indicates that their reading speed increased $60.72 \%$ after the training sessions; without using the OVP. The result also shows when the participants were training their eye movements to fixate on the OVP, they could recognise words faster without having the OVP for eye control. Comparing reading performance between day 1 and day 7 training with the OVP on word recognition, students reading performance increases $64.12 \%$. NPS survey was used to ask their satisfaction with the product. The result shows the NPS score equals to 33 meaning the application is doing some good and the users are willing to recommend the application to their friends.

This study focuses on an application development and a pilot study on a word reading recognition. Much work needs to be done in the future. Testing should be done with more participants. The story reading part should be also used in a test to examine after children were trained to locate their eyes on the OVP of the word reading, whether their reading performance is also improved.

\section{Acknowledgment}

I am grateful to Witayanont School, Bangkok for recruiting the participants. I also want to thanks to Phuwamet Pornratworaphat and Worrapol Tieankhajorn for their great collaborators. Finally, many thanks to Bussarin Thosen and Fareeda Wanichayakorn for being a wonderful research assistant.

\section{References}

[1] Office of basic education commission. Basic education core curriculum of Thai language in 2008. Retrieved April 26, 2021 from https://www.obec.go.th/

[2] Country meters. Thailand population. Literacy of population. Retrieved April 26, 2021 from https://countrymeters.info/en/Thailand\#literacy

[3] Schleicher Andreas. PISA 2018: Insights and Interpretations. OECD Publishing, France, 2019, 64 pages.

[4] Scholar Within. Average Reading Speed by age grade level. Retrieved May 4, 2021 from $\mathrm{https} / / /$ scholarwithin.com/average-reading-speed\#

[5] Linden, Lotje \& Vitu, Françoise. On the optimal viewing position for object processing. Attention Perception \& Psychophysics, 2015. pp. 602-617.

[6] Andrew, Ben, Paul, Susana. Optimizing the viewing position of words increases reading speed in patients with central vision loss. Investigative Ophthalmology \& Visual Science June 2015, Vol.56, Issue 7.

[7] Royal Institute Dictionary. 2008. The principles of using punctuation marks and other symbols. Using a space in Thai written. Retrieved April 24, 2021 from https://www.orst.go.th/iwfm_search.asp

[8] Glenn Slayden, Mei-Yuh Hwang, and Lee Schwartz. 2010. Large-Scale Thai Statistical Machine Translation. Microsoft Technical Report MSR-TR-2010-41. Redmond: Microsoft Corporation.

[9] Kasisopa, Benjawan \& Reilly, Ronan \& Luksaneeyanawin, Sudaporn \& Burnham, Denis. Child Readers' Eye Movements in Reading Thai. Vision research, 2016. 123. 10.1016/j.visres.2015.07.009.

[10] Lehtimäki, Taina \& Reilly, Ronan. Improving Eye Movement Control in Young Readers. Artif. Intell. Rev.24. November, 2005, pp.477-488. 